\title{
Lessons from Umbilical Vein Varix
}

\author{
K Aparna Sharma ${ }^{1}$, Harpreet Kour Isher ${ }^{2}$, Vatsala Dadhwal ${ }^{3}$, Anubhuti Rana ${ }^{4}$, Abhijeet Kumar ${ }^{5}$
}

\begin{abstract}
Umbilical vein varix is an uncommon vascular anomaly. The varix may be the initial presentation of an underlying congenital portosystemic shunt adversely affecting the fetus as we demonstrate in this case. To be able to label a varix as an isolated finding necessitates a thorough evaluation of the fetal venous system as well. Serial ultrasound of the fetus can help in detecting changes in the varix, thrombosis, growth restriction, as well as heart failure if there is another underlying venous anomaly. Early postnatal imaging is essential to exclude other associations to optimize the neonatal outcome. The prognosis is good if it is an isolated finding.

Keywords: Portosystemic shunts, Umbilical vein, Varix.

International Journal of Infertility and Fetal Medicine (2020): 10.5005/jp-journals-10016-1201
\end{abstract}

\section{BACKGROUND}

Fetal intra-abdominal umbilical vein varix (FIUVV) is an uncommon finding on ultrasound. The prognosis is generally good in isolated FIUVV. We present a case of FIUVV with progressive deterioration of umbilical artery Dopplers and dilatation of systemic veins followed by a postnatal revelation of an intrahepatic portosystemic shunt.

\section{Case Description}

A 29-year-old G2P1L1 lady was referred at 26 weeks with a diagnosis of FIUVV. This was an unsupervised pregnancy with no prior aneuploidy screening. Gestational diabetes mellitus was detected at 27 weeks and controlled on diet. She had one healthy child aged 2 years.

On ultrasound, a dilated anechoic intra-abdominal extrahepatic cystic structure measuring $1.57 \times 1.50 \mathrm{~cm}$ was seen just to the right of the intra-abdominal portion of umbilical vein, in continuity with it (Fig. 1). Color Doppler showed venous waveform with no turbulence. Intrahepatic measurement of the umbilical vein was $0.6 \mathrm{~cm}$. Umbilical artery and ductus venosus Dopplers were normal. Fetal echocardiography revealed normal fetal cardiac anatomy with cardiothoracic ratio of $50 \%$. No soft markers of aneuploidy were identified on ultrasound. She was referred for genetic counseling and offered amniocentesis, which was declined.

The fetal growth was found to be appropriate for gestational age and weekly scans were done to monitor for variceal changes. Two weeks later, mild tricuspid regurgitation (TR) with mildly dilated right atrium, right ventricle with a prominent brachiocephalic vein (Fig. 2A) was noted. At $31+4$ weeks, FIUVV increased to $1.8 \times 1.9 \mathrm{~cm}$; a dilated inferior vena cava (IVC) and hepatic veins (Fig. 2B) were noted with turbulence in IVC besides a dilated right heart with a myocardial performance index of 0.50 . Umbilical artery showed reversed end diastolic flow (REDF); ductus venosus could not be visualized at this point. There was no hydrops. After antenatal steroids, an average for gestational age, male baby weighing, 1,560 g, with Apgar score 5.7, was delivered by caesarean at $31+6$ weeks due to REDF.

\section{Postnatal Course}

Postnatal echocardiography detected a 7-mm ostium secundum atrial septal defect (ASD). Ultrasound abdomen of the neonate
${ }^{1-4}$ Department of Obstetrics and Gynaecology, All India Institute of Medical Sciences, New Delhi, India

${ }^{5}$ Department of Obstetric and Gynecology, Base Hospital, New Delhi, India

Corresponding Author: Harpreet Kour Isher, Department of Obstetrics and Gynaecology, All India Institute of Medical Sciences, New Delhi, India, Phone: +91 8283841374, e-mail: harpreetsukhija1@yahoo.com

How to cite this article: Sharma KA, Kour Isher H, Dadhwal V, et al. Lessons from Umbilical Vein Varix. Int J Infertil Fetal Med 2020;11(1): 30-32.

Source of support: Nil

Conflict of interest: None

initially showed a dilated IVC, patent DV, with suspicion of an arteriovenous (AV) fistula between hepatic artery and portal vein. Two weeks later, a dilated left portal vein measuring $\sim 5 \mathrm{~mm}$ and dilated right hepatic vein with a communication between the two with shunt fraction $<50 \%$ was found on ultrasound and a diagnosis of congenital intrahepatic portosystemic shunt was made. The shunt was reconfirmed on CT angiogram done at 4 weeks of life. Blood karyotype confirmed trisomy 21. Initial concerns of respiratory distress, congestive heart failure, and

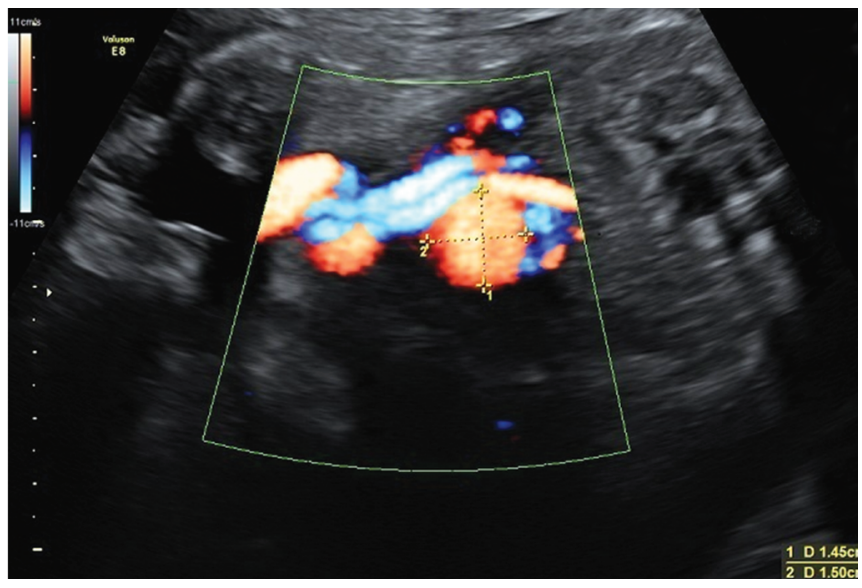

Fig. 1: Umbilical vein varix

(0) The Author(s). 2020 Open Access This article is distributed under the terms of the Creative Commons Attribution 4.0 International License (https://creativecommons. org/licenses/by-nc/4.0/), which permits unrestricted use, distribution, and non-commercial reproduction in any medium, provided you give appropriate credit to the original author(s) and the source, provide a link to the Creative Commons license, and indicate if changes were made. The Creative Commons Public Domain Dedication waiver (http://creativecommons.org/publicdomain/zero/1.0/) applies to the data made available in this article, unless otherwise stated. 

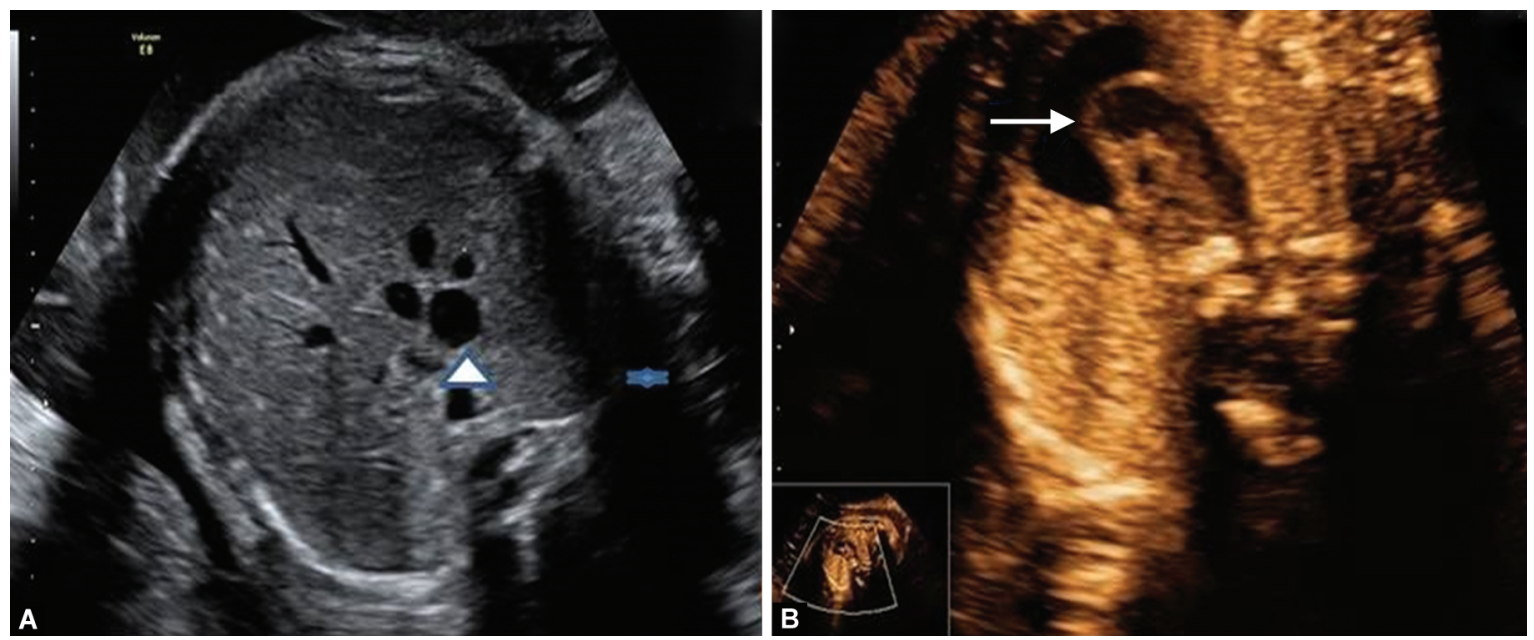

Figs 2A and B: (A) Dilated IVC (white arrow head) surrounded by prominent three hepatic veins; (B) Dilated brachiocephalic vein (arrow)

hyperbilirubinemia were managed and baby was discharged after 5 weeks with a plan for conservative management awaiting spontaneous closure of the shunt. Currently, the baby is 3 months old and under follow-up.

\section{Discussion}

The unsupported extrahepatic intra-abdominal portion of the umbilical vein is postulated to form a varix in conditions causing increased umbilical venous pressure. ${ }^{1}$ Fetal intra-abdominal umbilical vein varix has a reported incidence of $0.4-1.1 / 1,000$ fetuses. The technique of measuring a FIUVV and diagnostic criteria have been defined by Nyberg $^{2}$ and were applied here with the FIUVV measuring $>9 \mathrm{~mm}$, being $>50 \%$ of the diameter of the intrahepatic segment of the umbilical vein.

Beraud et al. in his review concluded that the three complications likely to occur with FIUVV are fetal demise, growth restriction, and variceal thrombosis with an overall frequency of $10 \%$, necessitating fetal surveillance. Fetal intra-abdominal umbilical vein varix may be associated with other anomalies in about $29-35 \%$ of cases; a detailed fetal anomaly scan and echocardiography are pertinent. ${ }^{3-5}$ Chromosomal abnormalities have been found in $6 \%$ of cases of FIUVV, commonly trisomy 21,18 , and triploidy. 3 ,6 Bas-Lando in his series of 24 patients with isolated FIUVV did not report any chromosomal anomaly. ${ }^{2}$ In this case, the fetus was under weekly ultrasound surveillance from 26 weeks. The increase in FIUVV size, dilated IVC, and right heart were suggestive of increased preload. Increase in size of FIUVV has been reported in about $28 \%$ cases. $^{4,5}$ This evolution of the changes in the vascular system of the fetus in retrospect could be attributed to the intrahepatic congenital porto-systemic shunt (CPSS), which was diagnosed postnatally. The CPSS may have led to the initial presentation as FIUVV, which could later progress to right heart failure.

Congenital portosystemic shunts are very rare vascular anomalies; only $10 \%$ are diagnosed prenatally. There are primarily two types of CPSS: extrahepatic and intrahepatic. Extrahepatic CPSS are more commonly associated with multiple anomalies, especially cardiovascular, than the intrahepatic CPSS, which may be associated with chromosomal anomalies as in this case. ${ }^{7}$ These abnormalities of the fetal venous circulation could interfere with liver perfusion leading to fetal growth restriction ${ }^{8}$ and neonatal problems as hyperbilirubinemia, elevated ammonia levels, galactosemia, and heart failure. These shunts are unlikely to cause symptoms if the shunt ratio is $<30 \%$ and are expected to close spontaneously in most cases by 1-2 years of age; otherwise, they need surgical intervention to prevent long-term complications as portopulmonary hypertension, hepatic tumors, endocrine dysfunction, and neurological deterioration., 7

Lee, in his large series of 121 cases of FIUVV, reported no fetus with chromosomal anomaly or a portosystemic shunt. ${ }^{10}$ Recently, Kawamura reported the coexistence of a portosystemic shunt with umbilical vein varix. ${ }^{11}$

\section{Conclusion}

Through this case, the lessons highlighted are:

- Detailed anomaly ultrasound, echocardiography, along with a critical examination of the fetal venous system and karyotype are essential before labelling FIUVV as isolated.

- Antenatal surveillance could be more intense starting at 26 weeks of gestation. Fetal echo could be repeated at 26-28 weeks to detect subtle signs as cardiomegaly and prominent veins and detect sudden-onset fetal heart failure, which could lead to sudden fetal demise.

- Extensive antenatal counseling of the couple is warranted explaining the possible risks associated with FIUVV from the point of detection, which should also include limitations in the current surveillance protocol based on the current available literature, as very few cases have been reported till now.

- Need for planning delivery in a tertiary set-up equipped with a pediatric cardiology unit to facilitate appropriate postnatal work-up, stabilization, and follow-up of the baby to improvise long-term outcome.

Though this is not a case series, it highlights the need to collect more data on the possibility of prenatal diagnosis of the coexistence of CPSS and FIUVV.

\section{Declaration}

Compliance with ethical standards.

\section{Ethical Approval}

This article does not contain any studies with human participants or animals performed by any of the authors. 


\section{References}

1. Nyberg D. Varix of the umbilical vein Nyberg DA, McGahan $J P$, Pretorius $\mathrm{DH}$, , et al. Diagnostic imaging of fetal anomalies. Philadelphia: Lippincott Williams \& Wilkins; 2003. pp. 114-115.

2. Bas-Lando M, Rabinowitz R, Samueloff $A$, et al. The prenatal diagnosis of isolated fetal varix of the intra-abdominal umbilical vein is associated with favorable neonatal outcome at term: a case series. Arch Gynecol Obstet 2013;288(1):33-39.

3. Byers BD, Goharkhay N, Mateus J, et al. Pregnancy outcome after ultrasound diagnosis of fetal intra-abdominal umbilical vein varix. Ultrasound Obstet Gynecol 2009;33(3):282-286.

4. Beraud E, Rozel C, Milon J, et al. Umbilical vein varix: importance of ante and post-natal monitoring by ultrasound. Diagn Interv Imaging 2015;96(1):21-26.

5. Rahemtullah A, Lieberman E, Benson C, et al. Outcome of pregnancy after prenatal diagnosis of umbilical vein varix. J Ultrasound Med 2001;20(2):135-139.
6. Fung $T Y$, Leung $T N$, Leung $T Y$, et al. Fetal intra-abdominalumbilical vein varix: what is the clinical significance? Ultrasound Obstet Gynecol 2005;25(2):149-154.

7. Papamichail M, Pizanias M, Heaton N. Congenital portosystemic venous shunt. Eur J Pediatr 2018;177(3):285-294.

8. Delle Chiaie L, Neuberger P, Von Kalle T. Congenital intrahepatic portosystemic shunt: prenatal diagnosis and possible influence on fetal growth. Ultrasound Obstet Gynecol 2008;32(2):233-235.

9. Uchino T, Matsuda I, Endo F. The long-term prognosis of congenital portosystemic venous shunt. J Pediatr 1999;135(2 Pt 1):254-256.

10. Lee SW, Kim MY, Kim JE, et al. Clinical characteristics and outcomes of antenatalfetal intra-abdominal umbilical vein varix detection. Obstet Gynecol Sci 2014;57(3):181-186.

11. Kawamura $\mathrm{H}$, Yashiro $\mathrm{K}$, Matsuo $\mathrm{S}$, et al. Visualization of intrahepatic portosystemic shunt and intra-abdominalumbilical vein varix using fetal ultrasonography with HDlive flow Imaging. J Med Ultrason 2020;47(1):143-145. DOI: 10.1007/s10396-019-00969-9. 